\title{
Solid-target seeded soft X-ray laser for short pulses and optical vortex amplification
}

Olivier Guilbaud, Alok-Kumar Pandey, Elsa Baynard, Irene papagiannouli, Fabrice Sanson, et al.

Olivier Guilbaud, Alok-Kumar Pandey, Elsa Baynard, Irene papagiannouli, Fabrice Sanson, Julien Demailly, Sophie Kazamias, Moana Pittman, Olivier Neveu, Bruno Lucas, Annette Calisti, Andrea Le Marec, Annie Klisnick, Olivier Larroche, F. Harms, G. Beaugrand, G. Dovillaire, Ph. Balcou, David Ros, "Solid-target seeded soft X-ray laser for short pulses and optical vortex amplification," Proc. SPIE 11886, International Conference on X-Ray Lasers 2020, 118860B (8 July 2021); doi: 10.1117/12.2593356 


\title{
Solid-target seeded soft $x$-ray laser for short pulses and optical vortex amplification
}

\author{
Olivier Guilbaud $^{\mathrm{a}^{*}}$, Alok-Kumar Pandey ${ }^{\mathrm{a}}$, Elsa Baynard ${ }^{\mathrm{a}}$, Irene Papagiannoulia ${ }^{\mathrm{a}}$, Fabrice Sanson ${ }^{\mathrm{a}, \mathrm{b}}$, \\ Julien Demailly ${ }^{\mathrm{a}}$, Sophie Kazamias ${ }^{\mathrm{a}}$, Moana Pittman ${ }^{\mathrm{a}}$, Olivier Neveu ${ }^{\mathrm{a}}$, Bruno Lucas ${ }^{\mathrm{a}}$, Annette \\ Calistic ${ }^{\mathrm{c}}$, Andrea Le Marec ${ }^{\mathrm{d}}$, Annie Klisnick ${ }^{\mathrm{d}}$, Olivier Larroche ${ }^{\mathrm{e}}$, F. Harms ${ }^{\mathrm{f}}$, G. Beaugrand ${ }^{\mathrm{f}}$, G. \\ Dovillaire ${ }^{\mathrm{f}}, \mathrm{Ph}$. Balcou $^{\mathrm{g}}$ and David Ros ${ }^{\mathrm{a}}$, \\ ${ }^{a}$ Laboratoire Irène Joliot-Curie, Université Paris-Saclay, UMR CNRS, Rue Ampère, Bâtiment

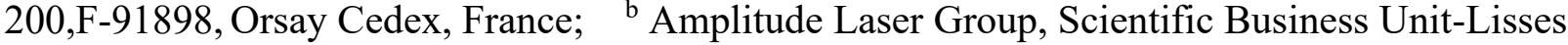 \\ Operations, 2/4 rue du Bois Chaland, 91090 Lisses, France; ' Aix-Marseille Université, CNRS, \\ Physique des Interactions Ioniques et Moléculaires, Centre Saint- Jérôme, F-13397 Marseille cedex \\ 20, France; ${ }^{\mathrm{d}}$ Institut des Sciences Moléculaires d'Orsay, Université Paris-Saclay , UMR CNRS \\ 8214, Rue André Rivière, Bâtiment 520, 91405 Orsay Cedex, France; ${ }^{e}$ CEA DAM DIF, 91297 \\ Arpajon, Cedex, France; ${ }^{\mathrm{f}}$ Imagine Optic, 18, rue Charles de Gaulle, 91400 Orsay, France.; ${ }^{\mathrm{g}}$ \\ Université Bordeaux-CNRS-CEA, CELIA, UMR 5107, Talence, France.
}

\begin{abstract}
We report temporal coherence measurement of solid-target plasma-based soft X-ray laser (XRL) in amplified spontaneous emission (ASE) mode. By changing the XRL pumping angle, we generate lasing at two-times higher electron density than the routine condition. A relatively shorter coherence time at a higher pumping angle indicates a clear spectral signature of higher electron density in the gain region. We probe the amplification dynamics of XRL in routine, and high electron density conditions to confirm gain-duration reduction resulting from ionization gating in the latter case. We also present recent results on the seeding of a vortex beam carrying orbital angular momentum (OAM) in XRL plasma. A small part of the high topological charge extreme ultraviolet (EUV) vortex is injected in XRL. These preliminary results suggest that the vortex seed indeed can be efficiently amplified. In the end, we propose a pathway towards the seeding of the complete vortex beam and wavefront characterization of the amplified beam.
\end{abstract}

Keywords: EUV, X-ray laser, X-ray interferometry, Coherence, high-harmonic generation, EUV vortex,

\section{INTRODUCTION}

The seeded operation of plasma-based soft x-ray laser (XRL) by high-order harmonics (HH) of the driving laser represents an important step forward for this field of research [1]. It has been demonstrated that some of the HH seed properties such as polarization state [1], and spatial coherence [2] can be transferred to the amplified beam. However, the HH pulse duration is expected to be strongly stretched due to the orders of magnitude spectral bandwidth difference between the seed and the amplifier [3]. Recovering as much of the original pulse duration is a subject of active research, and different paths have been suggested. One of them is to seek higher electron density into the lasing region [4]. Even if this approach is straightforward in principle, in practice, the resulting increase in the electron density gradient may be detrimental to the amplification.

Besides, in the recent past, there has been an increased interest in the generation and characterization of optical vortices carrying Orbital Angular Momentum [5]. Such beams have a transverse helical phase with $e^{i l \Phi}$ dependence around the beam propagation axis. This leads to an annular profile with null on-axis intensity due to central phase singularity: $l$ is the

*olivier.guilbaud@universite-paris-saclay.fr

International Conference on X-Ray Lasers 2020, edited by Davide Bleiner,

Proc. of SPIE Vol. 11886, 118860B - () 2021 SPIE · CCC code:

0277-786X/21/\$21 d doi: $10.1117 / 12.2593356$

Proc. of SPIE Vol. 11886 118860B-1 
number of $2 \pi$ phase shifts along the azimuthal coordinate $\Phi$ of the beam signifying the azimuthal mode index, also known as the topological charge. Moreover, high harmonic generation driven by optical vortices provides a straightforward way to extend the singular light field to extreme ultraviolet (EUV) spectral range [6-8]. During upconversion of the vortex driver of charge $l_{1}$, the momentum conservation imposes a linear upscaling of the topological charge with harmonic order [9]: $l_{q}=q l_{1}$, where $l_{q}$ is the topological charge of $q^{\text {th }}$ harmonic. In the context of XRL, the possibility of EUV vortex amplification in XRL-plasma is intriguing. Efficient amplification of circularly polarized HH seed, hence conservation of Spin Angular Momentum (SAM), has been demonstrated in a $\mathrm{Kr}^{8+}$ plasma amplifier [10]. However, the conservation of OAM during amplification in XRL-plasma is a fundamental question that remains unanswered. In general, the electron density gradient in the plasma amplifier affects the wavefront of the seeded beam. This issue can particularly be critical for the amplification of helically-phased vortex beams. Recent progress in soft X-ray wavefront metrology finally has permitted amplitude, phase, and modal content characterization of EUV vortices obtained through $\mathrm{HH}$ generation in rare gas $[8,11-12]$. Therefore, it is now possible to investigate whether the OAM carried by optical vortices is conserved during the amplification in XRL-plasma.

In this paper, we will first describe the successful amplified spontaneous emission (ASE) and seeded operation of XRL pumped in a configuration expected to double the electron density in the lasing region. The infrared pumping beam grazing angle $\Phi$ has been increased from its standard value $\Phi=22^{\circ}$ to $\Phi=32^{\circ}$, allowing the pump to penetrate deeper into the preformed plasma. The seeded operation has been achieved in both cases. For the high angle case, an off-axis injection has been performed to compensate for the density gradient refraction. Temporal coherence measurement of ASE-XRL and gain-probing investigation indicates two-times higher electron density in the lasing region for higher pumping angle configuration. In the second part, we will present our recent progress on the seeding of vortex beams carrying OAM in the XRL plasma amplifier.

\section{SEEDED SOFT X-RAY LASER PLATFORM}

A general schematic of the experimental setup is depicted in Fig. 1 [13]. The lasing medium comprises an elongated laserproduced plasma in which Ne-like Titanium ions are excited by the plasma hot electrons through transient collisional excitation [14]. The transient population inversion obtained between the $3 p-3 s(J=0-1)$ ionic transition corresponds to the lasing wavelength $\lambda=32.6 \mathrm{~nm}$. The lasing plasma is obtained in two steps [13, 15]. A frequency-doubled $400 \mathrm{~mJ} \mathrm{Q}-$ switched Nd YAG laser pulse of duration $10 \mathrm{~ns}$ is focused at normal incidence on a $4 \mathrm{~mm}$ Titanium target using a cylindrical lens to form a $5 \mathrm{~mm} \times 80 \mu \mathrm{m}$ focal line. Given pulse turns a thin layer of the Titanium target into an expanded, low ionization stage $(<\mathrm{Z}>\sim 5-6)$, moderate electron temperature $(\mathrm{Te} \sim 20 \mathrm{eV})$ preplasma. After an optimal delay of 4 ns following the temporal peak intensity of preplasma pulse, an intense near-infrared (IR) pump pulse with an energy of $\sim 1.2 \mathrm{~J}$ is line-focused on the preformed plasma using a spherical mirror of $500 \mathrm{~mm}$ focal length. The grazing-incidence pumping (GRIP) angle $\Phi$ is adjustable between $18^{\circ}$ and $34^{\circ}$. Owing to GRIP geometry, the pump energy is mostly absorbed at the turning point of its chief ray trajectory, corresponding to the electron density of $\mathrm{Np}=1.2 \times 10^{21} \mathrm{~cm}^{-3} \sin ^{2}(\Phi)[16]$. Therefore, an increase in $\Phi$ leads to deeper penetration of the pump energy into preplasma. Before the following study, we measured the ASE-XRL signal level for pumping angles $\Phi$ ranging from $18^{\circ}$ to $34^{\circ}$ by a step of $2^{\circ}$. For each GRIP angle, an optimum pump pulse duration $\mathrm{T}_{\mathrm{IR}}$ yielding maximum signal level is obtained.

For temporal coherence measurement, two configurations leading to a factor of two in the GRIP electron density were finally retained: (1) a routine condition $\left(\Phi=22^{\circ}\right.$; optimal pumping pulse duration $\left.\mathrm{T}_{\mathrm{IR}}=7 \mathrm{ps}\right)$ corresponding to a penetration density of $\mathrm{Np}=2 \times 10^{20} \mathrm{~cm}^{-3}$, and (2) a high GRIP angle configuration $\left(\Phi=32^{\circ}\right.$; optimal pumping pulse duration $\mathrm{T}_{\mathrm{IR}}=4$ ps) associated with the electron density $\mathrm{Np}=4 \times 10^{20} \mathrm{~cm}^{-3}$. Besides, to draw easier comparisons, for both configurations, the measurements have been also performed for the pulse duration of $\mathrm{T}_{\mathrm{IR}}=4 \mathrm{ps}$ and $\mathrm{T}_{\mathrm{IR}}=7 \mathrm{ps}$.

The residual preplasma, pumping, and $\mathrm{HH}$ driving pulses are filtered using a $300 \mathrm{~nm}$ thick $\mathrm{Al}$ filter. To measure the temporal coherence, the ASE-XRL beam is directed towards the wavefront-division interferometer set at $\sim 3.2 \mathrm{~m}$ from the source through a $45^{\circ}$ multilayer mirror acting as a spectral filter. Given interferometer enables the introduction of a variable path difference $\delta$ between the interfering beamlets without changing their overlapping conditions; its conception and working are detailed in [17]. The fringe visibility $\mathrm{V}$ averaged over multiple single-shot interferograms as a function of $\tau=$ $\delta / c$ under four experimental configurations previously described is presented in Fig. 2. 


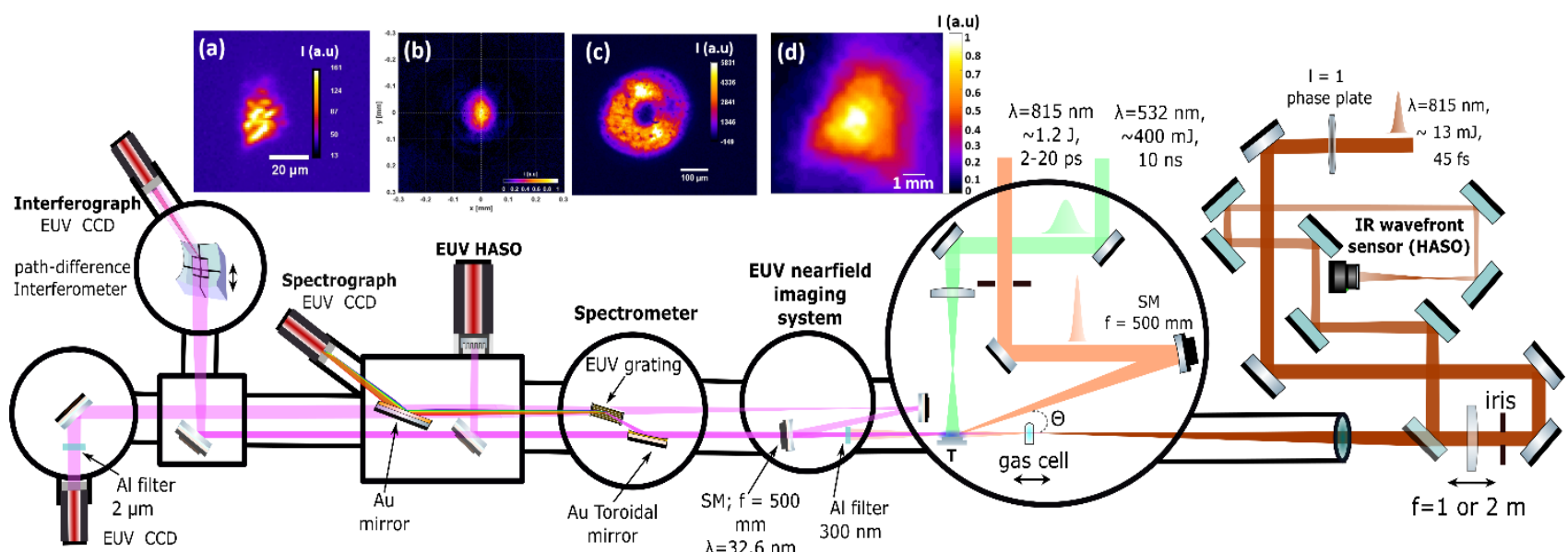

Fig. 1. Overview of the experimental setup. The preplasma is generated by focusing a frequency-doubled Q-switched Nd YAG laser pulse of 10 ns FWHM duration on the Titanium target using a cylindrical lens. High-energy pump pulse of adjustable duration (tunable between 2 to 20 ps) coming from Ti: Sapphire laser-chain is line-focused on the target using a spherical mirror (SM) of focal length 500 $\mathrm{mm}$. The pump grazing-incidence angle $\Phi$ can be adjusted under vacuum using motorized mirror mounts. The near-field intensity profile of the source is characterized using a high magnification monochromatic EUV (32.6 nm wavelength) imaging system. The inset figure (a) shows the near-field profile of ASE-XRL for $\Phi=32^{\circ}, \mathrm{T}_{\mathrm{IR}}=4 \mathrm{ps}, 4 \mathrm{~ns}$ peak-to-peak delay between the preplasma and pump pulse. To measure the temporal coherence, the XRL pulse is guided to a path-difference interferometer situated $\sim 3.2 \mathrm{~m}$ further from the source. Besides, the beamline is also equipped with wavefront sensors for near-IR (HASO4 FIRST; $800 \pm 50 \mathrm{~nm}$; Imagine Optic) and EUV to soft X-ray (EUV HASO; 40 to $4 \mathrm{~nm}$; Imagine Optic), permitting wavefront metrology in the stated spectral range. For seeded operation, the HH seed is generated by focusing an auxiliary low-energy infrared pulse of duration $\sim 40 \mathrm{fs}$ in an Argon filled gas-cell. The harmonic beam carrying orbital angular momentum is generated by inserting a segmented phase plate (HoloOr) in the HH driving beam. Inset figure (b) depicts the nearfield intensity profile of the Gaussian HH seed, whereas the nearfield intensity profile of the vortex seed is shown in (c). In (d), seeded-XRL far-field profile acquired on EUV CCD located $\sim 1.9 \mathrm{~m}$ from the source for $\Phi=32^{\circ}, \mathrm{T}_{\mathrm{IR}}=4$ ps is shown.

A relatively faster decline of the fringe visibility is observed for $\Phi=32^{\circ}$ as compared to $\Phi=22^{\circ}$ pumping angle for both the considered pumping pulse durations of $4 \mathrm{ps}$ and $7 \mathrm{ps}$. This behavior is consistent with a relatively higher electron density in the gain region that leads to a larger spectral bandwidth and hence, a smaller coherence time. Following the approach proposed by Le Marec et al [18, 19], a curve fitting procedure has been developed to perform a quantitative analysis of these results. Further details of this analysis can be found in [13]; only a summary of the said procedure will be presented here. It is well known that the fringe visibility $\mathrm{V}(\tau)$ is related to the Fourier transform of the laser spectral lineshape. Consequently, the width of $\mathrm{V}(\tau)$ or coherence time $\tau_{\mathrm{c}}$ is inversely proportional to the spectral bandwidth $\Delta v$. However, because of the stochastic structure of ASE pulse, and $\tau_{\mathrm{c}}$ being close to the ASE pulse duration $\tau_{\mathrm{xuv}}$, the visibility curve $\mathrm{V}(\tau)$ will deviate from this classical behavior at a large value of $\tau$, where its evolution will be governed by $\tau_{\mathrm{xuv}}$. Therefore, the average experimental visibilities corresponding to delays $-1 \mathrm{ps}<\tau<1 \mathrm{ps}$ are fitted with a first gaussian model from which values of coherence time $\tau_{\mathrm{c}}$ and bandwidth $\Delta v$ are extracted. These curves are displayed in red in Fig.

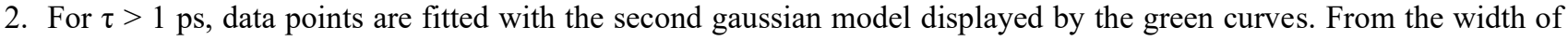
this curve, the pulse duration $\tau_{\text {pulse }}$ is obtained. The corresponding values of coherence time and pulse duration are displayed in the insets of Fig. 2.

The quantitative analysis confirms a relatively shorter coherence time ( a factor of 2 ) for the high-density scenario of $\Phi=$ $32^{\circ}$ when compared to $\Phi=22^{\circ}$ for both pump pulse durations. This is consistent with a two-times increase in electron density as the laser bandwidth is dominated by collisional homogeneous broadening for such XRL. For ASE XRL, the pulse duration is governed by the gain dynamics, which is inevitably influenced by the electron density in the gain region. Therefore, similar to the trend observed for coherence time, the deduced pulse duration $\tau_{\text {pulse }}$ reduces by a factor of two for $\Phi=32^{\circ}$ high-density case. 

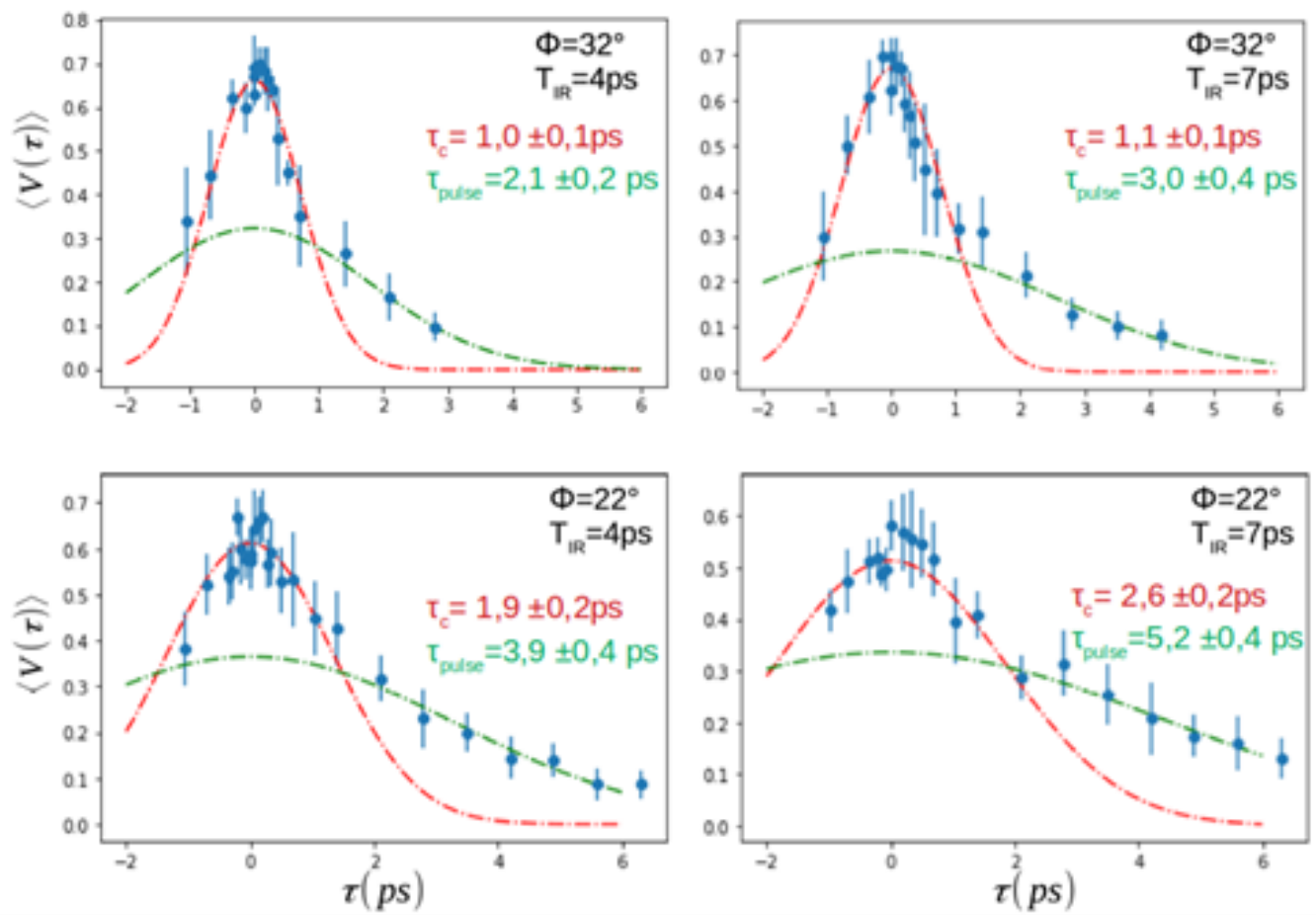

Fig. 2. Measured fringe visibility as a function of relative delay between the interfering beamlets for grazing incidence pumping angles $\Phi=32^{\circ}$ (top row) and $\Phi=22^{\circ}$ (bottom row) for pump pulse duration $\mathrm{T}_{\mathrm{IR}}=4$ ps and $7 \mathrm{ps}$ in the respective cases. For each delay, the data point is an average of the maximum visibility for 5 to 10 single-shot interferograms while the error bars represent the standard deviation. The duration of the pump pulse is varied by altering the distance between in-vacuum compressor gratings. In all the cases, the peak-to-peak delay between preplasma and pump pulse is $4 \mathrm{~ns}$.

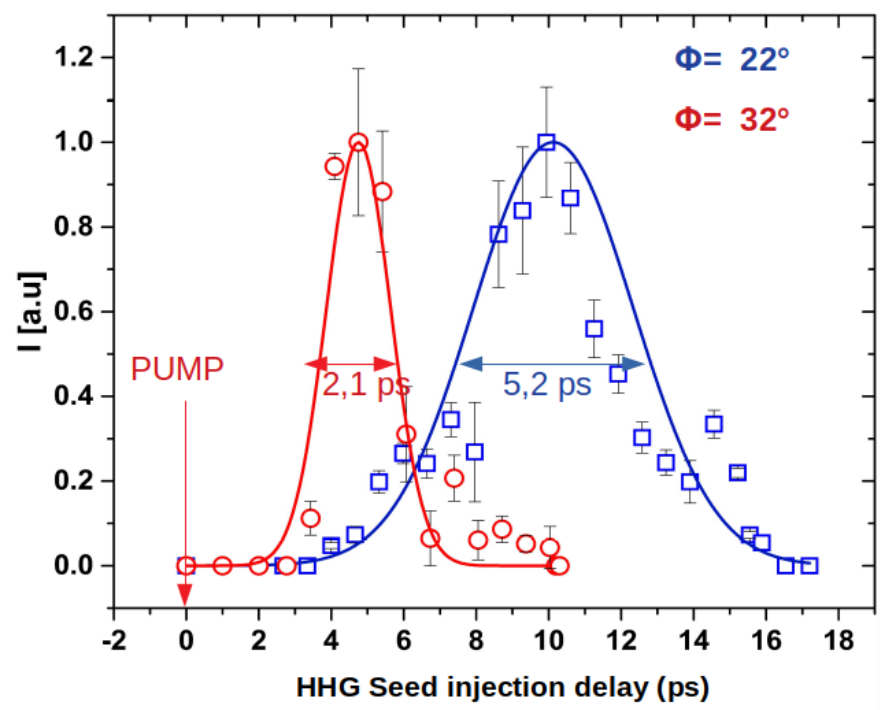

Fig. 3. Normalized seeded-XRL energy as a function of injection delays for low $\left(\Phi=22^{\circ} ; \mathrm{T}_{\mathrm{IR}}=7 \mathrm{ps}\right)$ and high $\left(\Phi=32^{\circ} ; \mathrm{T}_{\mathrm{IR}}=4 \mathrm{ps}\right)$ electron density amplifiers. The XRL plasma was seeded at an off-axis injection angle of $\sim 1^{\circ}$ for $\Phi=32^{\circ}$ to counter the refraction induced due to a higher electron density gradient in the latter case. The peak-to-peak delay between the preplasma and pump pulse is 4 ns for both cases. 
To assess the effect of higher electron density on the gain dynamics of XRL, we compare the $\tau_{\text {xuv }}$ values to the results of the XRL gain-probing experiment. Seeded operation of XRL for $\mathrm{T}_{\mathrm{IR}}=7 \mathrm{ps}$ at $\Phi=22^{\circ}$ and $\mathrm{T}_{\mathrm{IR}}=4 \mathrm{ps}$ at $\Phi=32^{\circ}$ has been achieved. An auxiliary low-energy $(\leq 13 \mathrm{~mJ})$ infrared beam of pulse duration $\sim 40 \mathrm{fs}$ is used to generate the HH seed in an Argon filled gas-cell. The seed source is placed $\sim 40 \mathrm{~mm}$ before the entrance of the plasma. The $25^{\text {th }}$ harmonic is spectrally, spatially, and temporally matched with the amplifier. For $\Phi=32^{\circ}$ configuration, the injection angle of the $\mathrm{HH}$ seed has been optimized to mitigate the refraction arising from the higher electron density gradient. The given injection angle was found to be $1^{\circ}$ higher as compared with $\Phi=22^{\circ}$ case. For a range of injection delays, an intense, and low divergence seeded beam dominating the diverging ASE beam, is obtained. The normalized seeded-XRL intensity as a function of seed injection delay is shown in Fig. 3 for both pumping angles $\Phi$. The temporal window during which seed is amplified is significantly shorter for $\Phi=32^{\circ}$ compared to $\Phi=22^{\circ}$ : amplification window at FWHM for the respective cases are $2 \pm 0.5$ ps and $4.7 \pm 0.5$ ps. The shorter amplification lifetime for a higher $\Phi$ value is again consistent with a larger electron density that leads to a faster collisional ionization gating [5]. Furthermore, the experimentally measured amplification lifetimes agree with the ASE pulse duration $\tau_{\text {pulse }}$ deduced from the temporal coherence analysis.

The presented experimental results indicate that the efficient lasing and seeded operation observed for $\Phi=32^{\circ}$ pumping configuration was indeed achieved at an electron density two times higher than the routine conditions. One-dimensional Bloch Maxwell simulations have been performed to predict the seeded-XRL pulse duration in both configurations [13]. For the $\Phi=22^{\circ}$ case, a final pulse duration of $\sim 1 \mathrm{ps}$ is expected, which agrees with the findings reported in [20]. At the higher pumping angle of $\Phi=32^{\circ}$, the amplified pulse is anticipated to have a duration of $\sim 500 \mathrm{fs}$. In conclusion, this straightforward approach is promising for the generation of sub-picosecond pulses. Moreover, the main drawback of the mandatory seed refraction compensation might be relaxed if this approach is applied to Ni-like Molybdenum, which is known to efficiently lase under similar pumping conditions, but at a shorter wavelength.

\section{AMPLIFICATION OF EUV VORTEX}

With the know-how of seeded-XRL operation [13, 15], and EUV-vortex wavefront metrology [11, 12], we recently attempted to inject high-charge EUV vortex in X-ray laser plasma. The primary interest is to answer the following fundamental question: does OAM carried by the vortex seed gets transferred to the amplified beam? Experimentally, the given study is particularly challenging: requiring EUV metrology tools, and precise spatial, temporal, spectral matching of the vortex seed to the amplifying XRL plasma.

To generate EUV vortex, a spiral phase plate designed for wavelength $815 \mathrm{~nm}$ (HoloOr) is inserted into the incoming Gaussian beam to impart a helical phase of unit topological charge. The resulting IR-vortex is apertured using an iris of diameter $\sim 20 \mathrm{~mm}$ and loosely focused by a $2 \mathrm{~m}$ focal length lens into a $15 \mathrm{~mm}$ long Argon-filled gas-cell at $\sim 12 \mathrm{mbar}$ pressure to generate $\mathrm{HH}$. The energy of the IR beam is $\sim 11 \mathrm{~mJ}$ after iris. The residual IR beam is filtered using a $300 \mathrm{~nm}$ thick Al filter. The $25^{\text {th }}$ harmonic (centered at $\lambda=32.6 \mathrm{~nm}$; spectrally matched to the lasing wavelength of Ne like Ti XRL) of the IR driver is spectrally selected using dielectric multilayer mirrors before being guided to different diagnostic tools. The previous experiment with these generation conditions has led to a harmonic signal level sufficiently high to perform single-shot wavefront characterization of EUV vortex [21]. In Fig. 4 (a), we show the farfield intensity profile of high topological charge EUV vortex $\left(l_{q}=q l_{1}\right.$ where q, the harmonic order is 25 , and $\left.l_{1}=1\right)$ reconstructed from single-shot Hartmanngram. The intensity profile at the exit of the gas-cell is acquired using a EUV imaging system and is shown in Fig. 1(c). An annular intensity profile is observed in nearfield as well as farfield. This annulus corresponds to a divergence of $\sim 5 \mathrm{mrad}$, much larger than the classical HH beam divergence $(<1 \mathrm{mrad})$.

Besides, one of the critical issues for vortex beam injection is the larger size of the seed in comparison to the amplifying cross-section of XRL. Fig. 4(b) shows the nearfield profile of ASE-XRL for GRIP angle $\Phi=22^{\circ}, T_{I R}=7$ ps is, whereas intensity profile of vortex seed at the exit plane of XRL is presented in Fig. 4(c). The gas-cell is placed $\sim 37 \mathrm{~mm}$ before the entrance of $3 \mathrm{~mm}$ long XRL plasma. As the nearfield EUV imaging system is configured to image the exit plane of XRL, Fig. 4(c) corresponds to vortex intensity profile $\sim 40 \mathrm{~mm}$ after the generation medium. Furthermore, the gas-cell is located $\sim 40 \mathrm{~mm}$ before the waist of the driving IR-vortex beam to optimize the signal level. To compensate for the size difference between XRL and vortex seed, an ideal alternative is the demagnification of the seed using additional optics to match the size of the amplifying plasma. However, this also necessitates a beam transport system capable of guiding a strongly diverging amplified beam to various diagnostics. In the recently held first experimental campaign, instead of refocusing, 
we opted to inject only a part of the vortex beam into XRL plasma. The spatial superposition of the two was ensured using the nearfield imaging system. In Fig. 4(d), we show the nearfield image when a part of the vortex seed is injected and amplified by the XRL. The position of the target surface, OAM seed, and the amplified zone are marked in the said figure. Ten consecutive harmonic shots are accumulated to keep the track of the part of the seed which is injected; the XRL is operated in single-shot mode hence, only the first harmonic shot is properly timed and amplified in the XRL plasma. The nearfield image shown in Fig. 4(d) is acquired after removing the residual driving beams by a metallic filter ( $300 \mathrm{~nm}$ thick $\mathrm{Al})$. Strongly saturated CCD pixels indicate an efficient coupling and amplification of the vortex seed. Moreover, when imaged with a higher attenuation ( 2 um Al filter), opposed to the speckled structure of ASE XRL, a smooth spatial profile is observed (not shown here). While initial results presented here are highly encouraging, further data analysis is needed to conclude if the helical wavefront of the vortex seed is retained during the amplification process. The findings after further analysis of the acquired data will be reported in a forthcoming article.
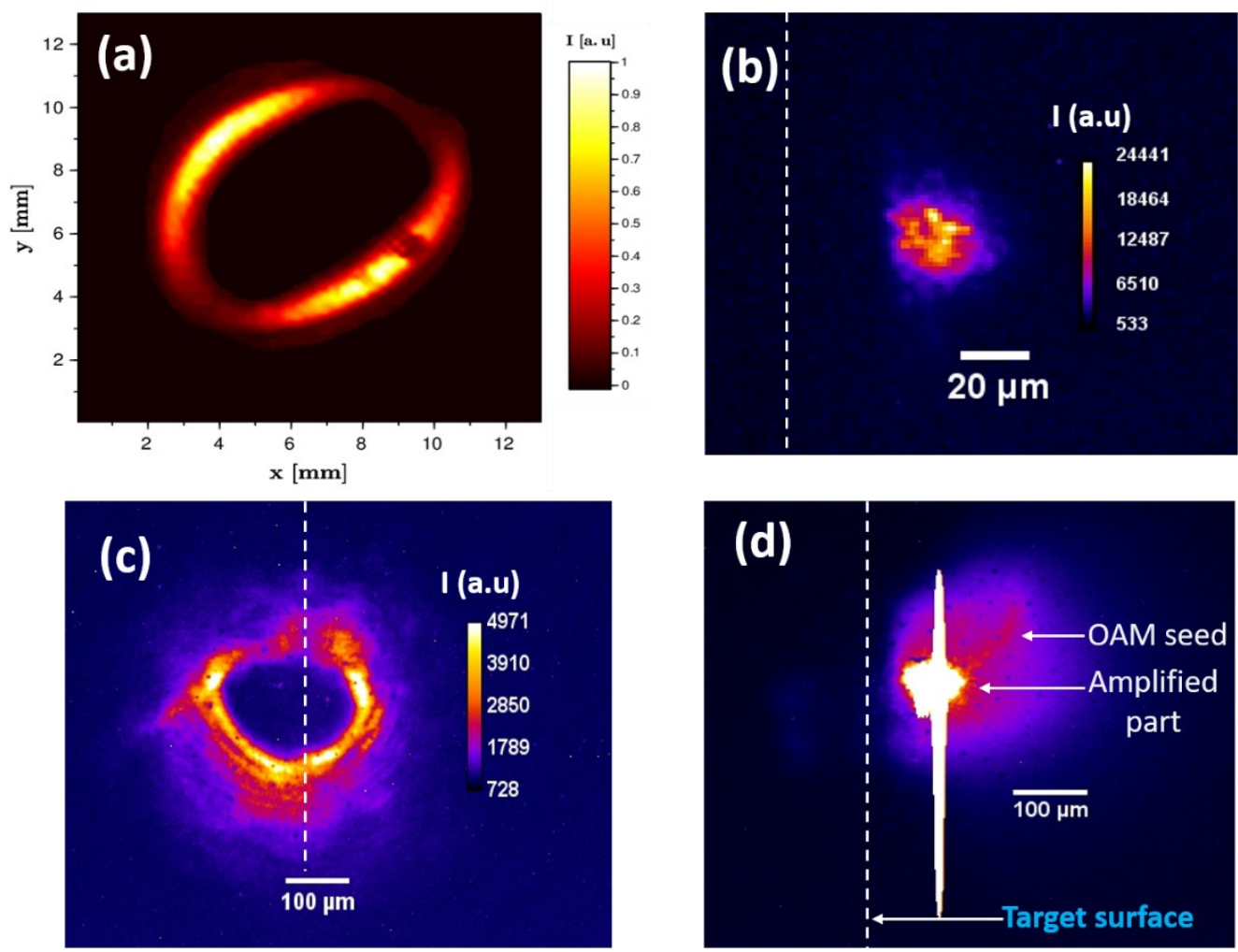

Fig. 4. Injection of vortex beam carrying $l=25$ OAM in XRL-plasma. (a) Farfield intensity profile of vortex beam reconstructed from single-shot Hartmanngram. (b) Nearfield profile of ASE-XRL is acquired using a EUV imaging system with a magnification factor of $\sim 10$. The vertical dashed line represents the position of the target surface. (c) The intensity profile of the vortex seed at the XRL exitplane. The gas-cell in which high-harmonics are generated is placed $\sim 40 \mathrm{~mm}$ before the exit plane XRL. The vertical dashed line marks the position of Ti Target during seeding. (d) As the size of the vortex seed is much larger than that of XRL (comparing (b) and (c)), only a part of the seed is injected and amplified in XRL plasma. The position of the OAM seed, target surface, and region of amplification are marked in (d). The image is acquired after filtering the residual driving beam with a $300 \mathrm{~nm}$ thick Al filter. Efficient amplification of a part of the vortex seed is evident from saturated CCD pixels. In this last image, 10 consecutive harmonic shots are accumulated with the only first one being amplified by the XRL. Multiple shots of harmonic are accumulated to track the part of the vortex seed that is amplified.

In summary, the seeded operation was not affected by the lower seed power per unit of the surface due to the larger size of the vortex beam at the entrance of the XRL-amplifier. Efficient amplification was also achieved for different positions of OAM harmonic annulus and XRL-pupil (top, bottom, left-right). In the upcoming experimental campaign, the vortex seed will be generated in a separate chamber and tightly focused on the entrance of the XRL plasma utilizing a combination of spherical and plane multilayer mirrors. The design and preliminary test show that the energy loss due to the reflectance 
of the optics will be compensated by the concentration of the vortex beam on a smaller area, leading to a seed intensity similar to the one obtained in this preliminary demonstration.

\section{CONCLUSIONS}

In conclusion, we have explicitly concentrated on the temporal coherence properties of the solid-target plasma-based XRL in experimental conditions that are expected to yield a factor of two in the electron density in the gain region. Through the coherence measurement, a clear spectral signature of higher electron density in the gain region has been observed and reported: a relatively shorter coherence time and hence, a larger spectral width for $\emptyset=32^{\circ}$ when compared to $\emptyset=22^{\circ}$. At higher electron density, the refraction is expected to be a challenge. Nonetheless, through off-axis injection, we have successfully seeded such a high-density amplifier. This is an encouraging result for further attempts with, for example, Nilike Mo lasing at a shorter wavelength. Furthermore, for metal-target amplifiers, for the first time to our knowledge, we demonstrate a reduction of amplification duration resulting from collisional ionization gating through a controlled increase of the electron density in the gain region. In the latter part of the article, we have presented our first result on the amplification of a high topological charge vortex beam in XRL plasma. With a prudent spatial superposition of the vortex seed and XRL amplifier, we opted to inject a part of the vortex and have observed a strong amplification. In the upcoming study, it is intended to demagnify the vortex seed to match the size of the seed and amplifying the cross-section of XRL.

A EUV Hartmann sensor will then be used to characterize the amplitude and phase of the amplified vortex beam.

\section{REFERENCES}

[1] Zeitoun, P., Faivre, G., Sebban, S., Mocek, T., Hallou, A., Fajardo, M., Aubert, D., Balcou, P., Burgy, F., Douillet, D., Kazamias, S., De Lachèze-Murel, G., Lefrou, T., Le Pape, S., Mercère, P., Merdji, H., Morlens, A. S., Rousseau, J. P. and Valentin, C., "A high-intensity highly coherent soft X-ray femtosecond laser seeded by a high harmonic beam,”. Nature 431, 426-429 (2004).; Wang et al. Phys. Rev. Lett. 97, 123901 (2006).

[2] Wang, Y., Granados, E., Larotonda, M. A., Berrill, M., Luther, B. M., Patel, D., Menoni, C. S. and Rocca, J. J., "High-brightness injection-seeded soft X-ray laser amplifier using a solid target," Phys. Rev. Lett. 97, 123901 (2006).

[3] Al'miev, I. R., Larroche, O., Benredjem, D., Dubau, J., Kazamias, S., Möller, C. and Klisnick, A., "Dynamical description of transient $x$-ray lasers seeded with high-order harmonic radiation through maxwell-bloch numerical simulations," Phys. Rev. Lett. 99(12), 123902 (2007).

[4] Depresseux, A., Oliva, E., Gautier, J., Tissandier, F., Nejdl, J., Kozlova, M., Maynard, G., Goddet, J. P., Tafzi, A., Lifschitz, A., Kim, H. T., Jacquemot, S., Malka, V., Phuoc, K. T., Thaury, C., Rousseau, P., Iaquaniello, G., Lefrou, T., Flacco, A., Vodungbo, B., Lambert, G., Rousse, A., Zeitoun, P. and Sebban, S., "Table-top femtosecond soft x-ray laser by collisional ionization gating," Nat. Photonics 9(12), 817-821 (2015).

[5] Wang, X., Nie, Z., Liang, Y., Wang, J., Li, T. and Jia, B., "Recent advances on optical vortex generation," Nanophotonics, 7(9), 1533-1556 (2018).

[6] Gariepy, G., Leach, J., Kim, K. T., Hammond, T. J., Frumker, E., Boyd, R. W. and Corkum, P. B., "Creating High-Harmonic Beams with Controlled Orbital Angular Momentum," Phys. Rev. Lett., 113, 153901 (2014).

[7] Zürch, M., Kern, C., Hansinger, P., Dreischuh, A. and Spielmann, Ch., "Strong-field physics with singular light beams,". Nature Phys 8, 743-746 (2012).

[8] Gauthier, D., Ribic, P. R., Adhikary, G., Camper, A., Chappuis, C., Cucini, R., DiMauro, L. F., Dovillaire, G., Frassetto, F., Géneaux, R., Miotti, P., Poletto, L., Ressel, B., Spezzani, C., Stupar, M., Ruchon, T. and Ninno, G. De., "Tunable orbital angular momentum in high-harmonic generation," Nat. Commun. 8, 14971 (2017).

[9] Hernández-Garcia, C., Picón, A., San Roman, J. and Plaja, L., "Attosecond extreme ultraviolet vortices from high-order harmonic generation," Phys. Rev. Lett. 111, 083602 (2013).

[10] Depresseux, A., Oliva, E., Gautier, J., Tissandier, F., Lambert, G., Vodungbo, B., Goddet, J. P., Tafzi, A., Nejdl, J., Kozlova, M., Maynard, G., Kim, H. T., Phuoc, K. T., Rousse, A., Zeitoun, P. and Sebban, S., "Demonstration of a circularly polarized plasma-based soft X-ray laser,” Phys. Rev. Lett. 115, 083901 (2015).

[11] Sanson, F., Pandey, A. K., Harms, F., Dovillaire, G., Baynard, E., Demailly, J., Guilbaud, O., Lucas, B., Neveu, O., Pittman, M., Ros, D., Richardson, M., Johnson, E., Li, W., Balcou, P. and Kazamias, S., "Hartmann wavefront 
sensor characterization of a high charge vortex beam in the extreme ultraviolet spectral range," Opt. Lett. 43(12), 2780-2783 (2018).

[12] Sanson, F., Pandey, A. K., Papagiannouli, I., Harms, F., Dovillaire, G., Baynard, E., Demailly, J., Guilbaud, O., Lucas, B., Neveu, O., Pittman, M., Ros, D., Richardson, M., Johnson, E., Li, W., Balcou, P. and Kazamias, S., "Highly multimodal structure of high topological charge extreme ultraviolet vortex beams," Opt. Lett. 45, 4790$4793(2020)$.

[13]Pandey, A. K., Papagiannouli, I., Sanson, F., Baynard, E., Demailly, J., Kazamias, S., Pittman, M., Neveu, O., Lucas, B., Le Marec, A., Klisnick, A., Calisti, A., Larroche, O., Ros, D. and Guilbaud, O., "Towards subpicosecond pulses from solid target plasma based seeded soft X-ray laser," Opt. Express 28, 28924-28941 (2020).

[14] Dunn, J., Osterheld, A. I., Li, Y., Nilsen, J. and Shlyaptsev, V. N., "Transient collisional excitation x-ray lasers with 1-ps tabletop drivers," IEEE J. Sel. Top. Quantum Electron. 5(6), 1441-1446 (1999).

[15] Guilbaud, O., Cojocaru, G. V., Li, L., Delmas, O., Ungureanu, R. G., Banici, R. A., Kazamias, S., Cassou, K., Neveu, O., Demailly, J., Baynard, E., Pittman, M., Marec, A. L., Klisnick, A., Zeitoun, P., Ursescu, D. and Ros, D., "Gain dynamics in quickly ionized plasma for seeded operated soft x-ray lasers," Opt. Lett. 40, 4775-4778 (2015).

[16] Keenan, R., Dunn, J., Patel, P. K., Price, D. F., Smith, R. F. and Shlyaptsev, V. N., "High-repetition-rate grazingincidence pumped x-ray laser operating at 18.9 nm," Phys. Rev. Lett. 94, 103901 (2005).

[17] Polack, F., Joyeux, D., Svatoš, J. and Phalippou, D., "Applications of wavefront division interferometers in soft x rays,” Rev. Sci. Instrum. 66(2), 2180-2183 (1995).

[18] Marec, A. L., Guilbaud, O., Larroche, O. and Klisnick, A., "Evidence of partial temporal coherence effects in the linear autocorrelation of extreme ultraviolet laser pulses," Opt. Lett. 41, 3387-3390 (2016).

[19] Marec, A. L., Larroche, O. and Klisnick, A., "Linear autocorrelation of partially coherent extreme-ultraviolet lasers: a quantitative analysis," Opt. Lett. 42, 4958-4961 (2017).

[20] Wang, Y., Berrill, M., Pedaci, F., Shakya, M. M., Gilbertson, S., Chang, Z., Granados, E., Luther, B. M., Larotonda, M. A. and Rocca, J. J., "Measurement of 1-ps soft-x-ray laser pulses from an injection-seeded plasma amplifier," Phys. Rev. A 79(2), 023810 (2009).

[21] Pandey, A. K., Sanson, F., Guilbaud, O., Papagiannouli, I., Harms, F., Dovillaire, G., Baynard, E., Demailly, J., Lucas, B., Neveu, O., Pittman, M., Ros, D., Richardson, M., Johnson, E., Li, W., Balcou, P. and Kazamias, S., "Impure infrared vortex driven high harmonic generation," in OSA High-brightness Sources and Light-driven Interactions Congress (EUVXRAY, HILAS, MICS), L. Assoufid, P. Naulleau, M. Couprie, T. Ishikawa, J. Rocca, C. Haefner, G. Sansone, T. Metzger, F. Quéré, M. Ebrahim-Zadeh, A. Helmy, F. Laurell, and G. Leo, eds., OSA Technical Digest (Optical Society of America, 2020), paper ETh3A.6 (2020). 